\title{
From PVE to HVE to fully laparoscopic rescue ALPPS: a case report of multidisciplinary management of giant HCC
}

\author{
Valentina Malerba', Andrea Benedetti Cacciaguerra', Giuseppe Zimmitti', Alberto Manzoni', Claudio \\ Sallemi ${ }^{2}$, Lorenzo Monfardini ${ }^{2}$, Massimo Graffeo $^{3}$, Claudio Bnà ${ }^{2}$, Giuseppe Natalini ${ }^{4}$, Alberto Zaniboni ${ }^{5}$, \\ Maria Cristina Montresor ${ }^{6}$, Claudio Codignola ${ }^{1}$, Marco Garatti ${ }^{1}$, Mohammed Abu Hilal ${ }^{1}$ \\ 'Department of General Surgery, Istituto Ospedaliero Fondazione Poliambulanza, Brescia 25124, Italy. \\ ${ }^{2}$ Department of Radiology, Istituto Ospedaliero Fondazione Poliambulanza, Brescia 25124, Italy. \\ ${ }^{3}$ Department of Internal Medicine, Gastroenterology and Endoscopy, Istituto Ospedaliero Fondazione Poliambulanza, Brescia \\ 25124, Italy. \\ ${ }^{4}$ Department of Anesthesia and Intensive Care, Istituto Ospedaliero Fondazione Poliambulanza, Brescia 25124, Italy. \\ ${ }^{5}$ Department of Oncology, Istituto Ospedaliero Fondazione Poliambulanza, Brescia 25124, Italy. \\ ${ }^{6}$ Department of Pathology, Istituto Ospedaliero Fondazione Poliambulanza, Brescia 25124, Italy.
}

Correspondence to: Prof. Mohammed Abu Hilal, Department of Surgery, Poliambulanza Foundation Hospital Brescia, Via Bissolati n 57, Brescia 25124, Italy. E-mail: abuhilal9@gmail.com

\begin{abstract}
How to cite this article: Malerba V, Benedetti Cacciaguerra A, Zimmitti G, Manzoni A, Sallemi C, Monfardini L, Graffeo M, Bnà C, Natalini G, Zaniboni A, Montresor MC, Codignola C, Garatti M, Abu Hilal M. From PVE to HVE to fully laparoscopic rescue ALPPS: a case report of multidisciplinary management of giant HCC. Hepatoma Res 2021;7:19.
\end{abstract}

http://dx.doi.org/10.20517/2394-5079.2020.131

Received: 21 Oct 2020 First Decision: 14 Dec 2020 Revised: 3 Jan 2021 Accepted: 6 Jan 2021 Published: 9 Mar 2021

Academic Editor: Ho-Seong Han, Georgios Tsoulfas Copy Editor: Miao Zhang Production Editor: Jing Yu

\begin{abstract}
Different strategies have been used to induce preoperative liver hypertrophy and reduce the risk of postoperative liver failure. Those have included both radiological-interventional and surgical strategies, such as portal and hepatic vein embolization, 2-stage hepatectomy and associated liver partition with portal vein ligation for staged hepatectomy (ALPPS). Herein, we describe the case of a patient with a large right liver hepatocellular carcinoma not amenable to liver transplantation, with HBV-related chronic hepatitis and a small future liver remnant (FLR), who underwent a multistep approach to ensure a safe major laparoscopic resection with an adequate FLR.
\end{abstract}

Keywords: Portal vein embolization, hepatic vein embolization, rescue ALPPS, laparoscopic surgery, hepatectomy, hepatocellular carcinoma 


\section{INTRODUCTION}

Liver resection represents the most oncologically effective treatment for many hepatic tumors. When a liver resection is planned, a strict balance between oncological efficacy and surgical safety has to be respected. While oncological efficacy depends on the complete removal of the tumor, with negative surgical margins, surgical safety is based on a number of factors including the remnant liver volume (RLV) and its functional reserve, both essential to avoid one of the most feared and serious complications in liver surgery, namely post-hepatectomy liver failure (PHLF).

While the risk of developing PHLF is strictly related to the future liver remnant (FLR) volume $e^{[1-3]}$, integration between FLR volumetric data and liver parenchyma quality, commonly evaluated through the indocyanine green (ICG) clearance test ${ }^{[4-6]}$, is mandatory to achieve a correct estimation of the FLR functional reserve and of the risk for PHLF development. In this context, patients requiring a liver resection for hepatocellular carcinoma (HCC) represent a peculiar patient subgroup, because HCC development is in the majority of cases related to chronic liver disease affecting liver tissue quality, decreasing hepatic functional reserve, limiting the extension of feasible liver resection, and increasing the FLR volume required to perform a safe liver resection ${ }^{[7]}$.

Among many strategies aiming to optimize the FLR volume for patients requiring a major liver resection, preoperative portal vein embolization (PVE) is the most popular and validated ${ }^{[8-11]}$. More recently, the associated liver partition and portal vein ligation for staged hepatectomy (ALPPS) procedure has been proposed for patients with a very small FLR volume or a PVE failure ${ }^{[12]}$. ALPPS was initially criticized for the initial suboptimal short term results, but later, following progressive methodologic variations and better patient selection, it proved to be safe and effective ${ }^{[13]}$. Moreover, the most recent reports have highlighted the potential role of hepatic vein embolization (HVE), synchronous or subsequent to PVE, in inducing additional parenchymal hypertrophy to further increase the pool of patients that may benefit from surgical treatment ${ }^{[14]}$.

We herein report a case where the sequential adoption of all the aforementioned approaches was necessary to allow a radical resection of a large right liver HCC in a patient affected by chronic liver disease and with a small FLR.

\section{CASE REPORT}

In March 2020, a 72-year-old obese $\left(B M I=34.48 \mathrm{~kg} / \mathrm{m}^{2}\right)$ male, complaining of right upper quadrant pain, was diagnosed with an $11-\mathrm{cm}$ tumor in the right liver on abdominal ultrasound. On subsequent contrastenhanced computed tomography (CT), the lesion, approximately $10 \mathrm{~cm} \times 11 \mathrm{~cm} \times 9 \mathrm{~cm}$ in size, was located very close to the right Glissonian pedicle and showed enhancement in the arterial phase and washout in the portal and delayed phases [Figure 1]: such findings were consistent with a diagnosis of large right liver HCC. The patient had a history of asymptomatic hepatitis B. All the clinical findings were normal with no signs of cirrhosis. His tumour markers including $\alpha$-fetoprotein and carcinoembryonic antigen were normal. Other laboratory tests showed: HBsAg (+), HBcAb (-), HBV DNA $251 \mathrm{IU} / \mathrm{mL}$, AST 76 U/L (normal level: < 35), ALT $68 \mathrm{U} / \mathrm{L}$ (normal level < $60 \mathrm{U} / \mathrm{L}$ ) and GGT $74 \mathrm{U} / \mathrm{L}$ (normal level: < $70 \mathrm{U} / \mathrm{L}$ ), PLT $238 \times 10^{9} / \mathrm{L}$ (normal level 130-459 × 10//L), albumin 4.4 g/dL (normal level: 3.1-5.2 g/dL), bilirubin $0.8 \mathrm{mg} / \mathrm{dL}$ (normal level: < $1.2 \mathrm{mg} / \mathrm{dL}$ ). Colonoscopy and gastroscopy did not show any pathological findings.

The case was presented at our multidisciplinary tumor board (MDT), and a right hemi-hepatectomy was indicated. However, due to a small FLR volume (26\%), a right PVE was recommended and was performed on day 1. 

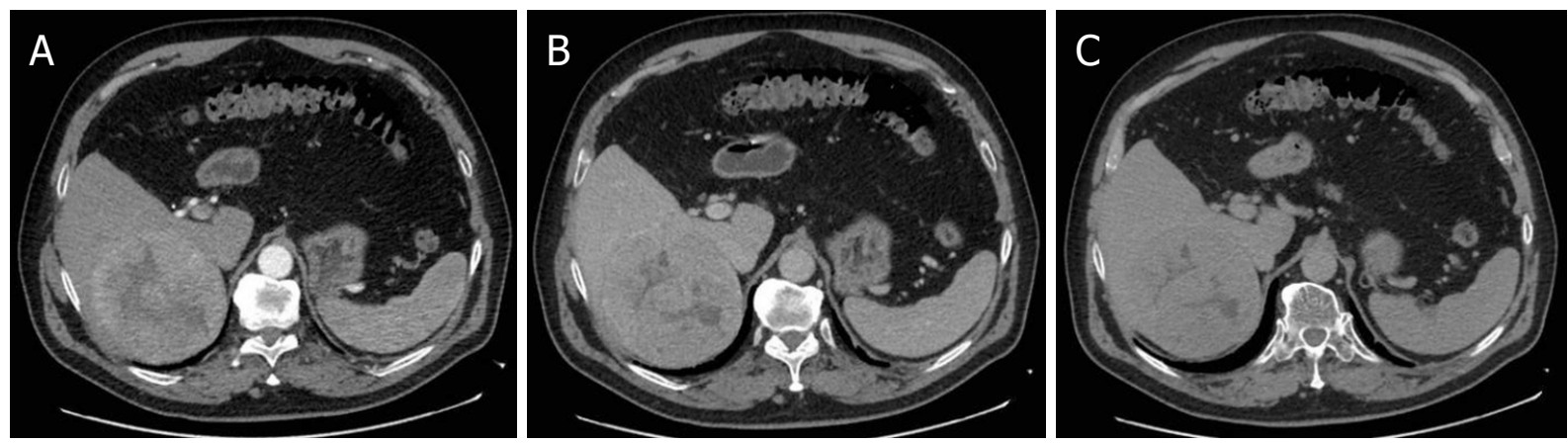

Figure 1. Our patient's first contrast-enhanced computed tomography showing the arterial enhancement $(A)$ of the liver lesion and its washout in the portal (B) and delayed (C) phases.

Five weeks later, CT scan volumetry revealed a total liver volume increase, from 26 to $34 \%$, but the patient's ICG test showed a retention rate at $15 \mathrm{~min}(\mathrm{R} 15)=19.5 \%$ (normal value $<10 \%)$ and a plasma disappearance rate $(\mathrm{PDR})=10.9 \%$ (normal value $>18 \%-24 \%$ per $\mathrm{min})$. A little growth of the lesion was also noticed $(12 \mathrm{~cm} \times 11 \mathrm{~cm} \times 10 \mathrm{~cm})$. CT and ICG test were repeated 8 weeks after right PVE but did not show any improvement. Given these findings, the MDT recommended a right HVE, which was performed on day 53 with ab Amplatzer ${ }^{\mathrm{TM}}$ vascular plug. After 6 weeks, CT volumetry showed a small increase in FLR volume (35\%) but a persistent suboptimal ICG clearance test, with R15 $=16 \%$ and PDR $=12.2 \%$.

Taking into account the persistently altered ICG test results, after re-discussion of the clinical case at MDT, it was decided to attempt, as a final salvage approach to obtain a further increase in FLR volume, a fully laparoscopic rescue ALPPS, consisting of two steps. The first step, a laparoscopic partial liver transection (LPLT), was performed on day 102.

\section{Surgical technique of fully laparoscopic rescue ALPPS}

\section{Laparoscopic partial liver transection}

Following a careful explorative laparoscopy and intraoperative liver ultrasound scan peritoneal metastasis and additional intrahepatic localizations were excluded. The hepatic hilum was dissected, and the right hepatic artery (RHA) was closed with a bulldog clamp, allowing the identification of a demarcation line on the anterior hepatic surface between the right and left liver. Such line was confirmed using ICG near-infrared (NIR) fluorescence (using the imaging technology RUBINA ${ }^{\mathrm{TM}}$ Stortz). The parenchymal transection along this line was performed using a Harmonic ${ }^{\circ}$ device for the superficial planes and Cavitron Ultrasonic Surgical Aspiration $\left(\mathrm{CUSA}^{\circ}\right.$ ) for the deeper planes and was not pushed deeper than about $5 \mathrm{~cm}$ in the hepatic parenchyma. Venous branches and Glissonean pedicles were dissected and divided between metallic clips or Hem-O-Lok ${ }^{\circledR}$ clips depending on their size. The operating time was 160 minutes, and the estimated blood loss was $100 \mathrm{~mL}$. No intraoperative complication occurred.

Following this operation, the patient was offered water and liquids on postoperative day (POD) one, semisolid diet on POD 2, and starting on POD 3, a free light diet. According to his adherence to such oral nutrition protocol, endovenous liquids were gradually stopped. Despite the absence of postoperative complications and a rapid postoperative recovery, the patient was not discharged, and one week following the first surgery, a CT scan was performed showing a growth of the entire liver up to $2823 \mathrm{~cm}^{3}$ and a FLR of $1128 \mathrm{~cm}^{3}$ ( $40 \%$ of the total liver volume). The ICG test showed a normalization in liver function, with an R15 of $6.2 \%$ and a PDR of $18.5 \%$. Therefore, the plan for a laparoscopic ALPPS completion was confirmed. On day 113 , the patient underwent a laparoscopic right hepatectomy with middle hepatic vein preservation. 


\section{Laparoscopic rescue ALPPS completion}

The procedure started with a complete mobilization of the right liver, followed by the dissection of the hepatic pedicle and the division of the right hepatic artery between $3 \mathrm{Hem}-\mathrm{O}-\mathrm{Lok}^{\bullet}$ clips. At this point, the parenchyma transection already started during the first operation was completed under intermittent hepatic pedicle occlusion as described above. The transection line passed between the right and left liver in the lower hepatic segments but cranially was shifted in the anterior right section, with intraparenchymal identification and transection of the right hepatic vein. This was due to the preoperative right hepatic vein (RHV) embolization, with embolizing material extending up to a few centimeters from the RHV-inferior vena cava (IVC) confluence and with a potentially high risk of dealing with a short and fragile RHV stump in case of venous transection close to the IVC confluence. Thus, we felt that separating the RHV some centimeters proximal to the IVC confluence, thus shifting the transection line to the right anterior section, preserving a longer venous stump, and finally performing a partial right hepatectomy, was a safer and oncologically acceptable option.

This operation duration was 380 minutes and the estimated blood loss was about $600 \mathrm{~mL}$ [Video 1].

The postoperative course was uneventful, and the patient was discharged 5 days after the ALPPS completion. The histopathological examination revealed a $13-\mathrm{cm}$ moderately differentiated HCC ( $\mathrm{pT} 2 \mathrm{Nx})$, and the nontumoral liver parenchyma assessment revealed fibrosis without signs of cirrhosis. All resection margins were clear.

\section{DISCUSSION}

HCC represents the most common primary hepatic tumor ${ }^{[15]}$, and liver resection, after liver transplantation, is the most effective treatment for patients with $\mathrm{HCC}^{[16]}$. When a major hepatectomy is planned, the FLR volume needed to minimize the risk of PHLF should take into account the quality of the FLR parenchyma. For a patient with a normal liver parenchyma, an FLR volume of at least 20\%-25\% may be considered sufficient, but among patients with HCC, due to the frequent coexistence of chronic (in the majority of cases virus-related or exotoxic) hepatitis, the need for an FLR volume higher than $30 \%-35 \%$ is often required $^{[17]}$. Multiple strategies to induce an adequate FLR hypertrophy have been described ${ }^{[18]}$. The case we report herein of an obese patient with a large right liver HCC, with HBV-related chronic hepatitis and a small FLR volume, shows how a modular use and a multi-step integration of different liver remodelling techniques may lead to achieving the required FLR hypertrophy before major liver resection, even in a particularly challenging case.

PVE, representing the most commonly used procedure to induce FLR hypertrophy, has been reported to have a success rate between $75 \%$ and $96 \%{ }^{[19]}$ and has been associated with low incidence of PHLF and mortality ( 6 and $3.6 \%$, respectively ${ }^{[20]}$. This was the first procedure performed in this case per our usual protocol $^{[21,22]}$, with a suboptimal increase in future liver volume (from 26 to $34 \%$ ), but the ICG clearance test, subsequently performed, revealed a severely impaired liver function.

When PVE was followed with a right HVE, no significant improvement was seen. This is in contrast to the results from recent studies, suggesting that sequential HVE, achieved thanks to the centrilobular congestion within the embolized territory immediately following the procedure ${ }^{[23]}$, may help to increase FLR hypertrophy in case of insufficient growth after $\mathrm{PVE}^{[19,24]}$. It should be highlighted that evidence concerning such strategy is quite limited and refer to small series of patients, suggesting that more studies concerning this issue are needed to confirm the efficacy of such strategy. In the current case, right HVE was shown to be safe but achieved only a marginal increase in FLR volume (from 34\% to 35\%) and a minimal improvement in liver function. It is possible that a better outcome could have been achieved if the HVE was performed simultaneously with the PVE as described in an experimental study by Van Lienden et al. ${ }^{[25]}$. 


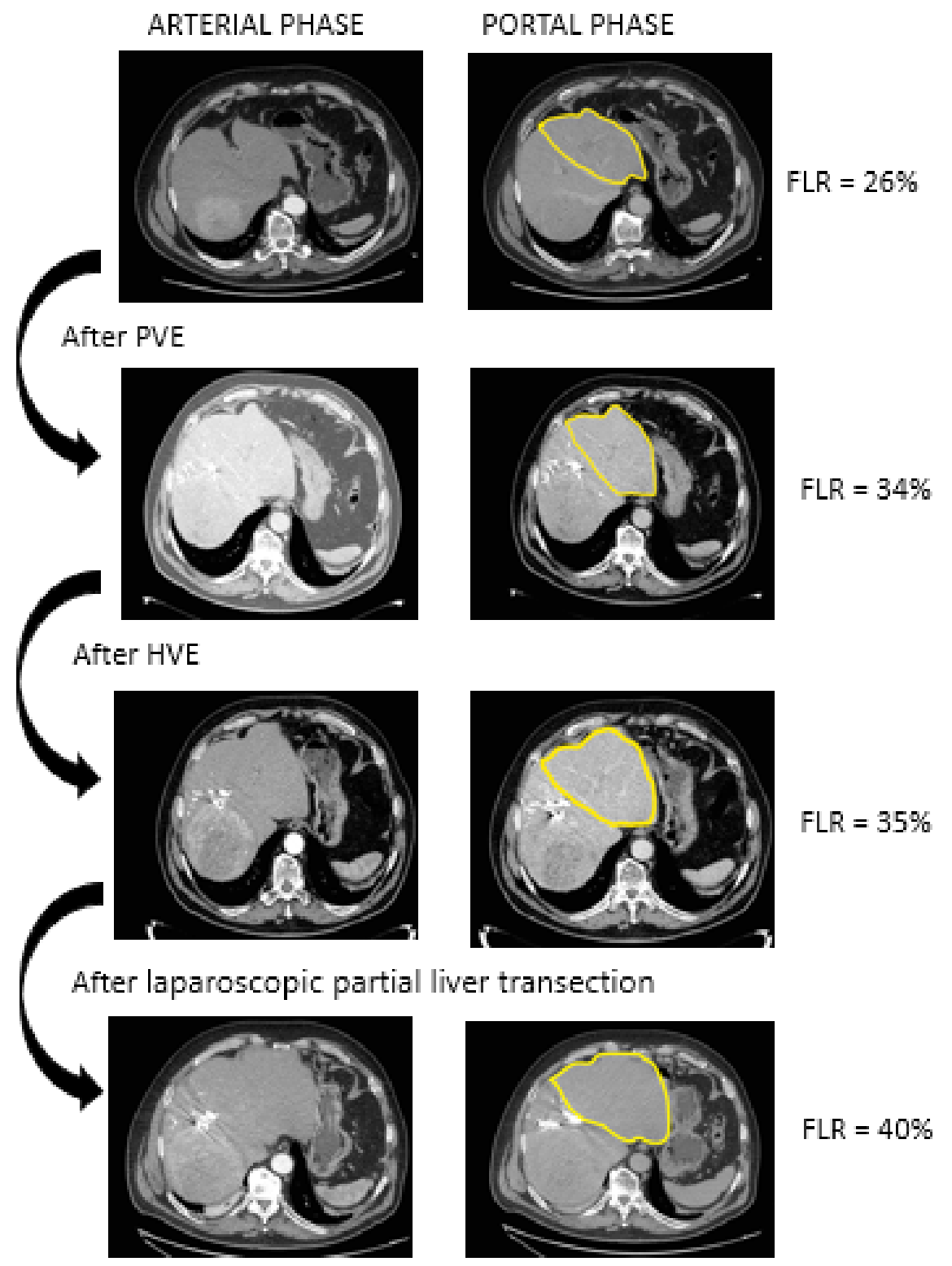

Figure 2. Series of contrast-enhanced computed tomography (in the arterial and portal phases) showing the development of the FLR after each procedure. FLR: future liver remnant; PVE: portal vein embolization; HVE: hepatic vein embolization.

At this point, although $35 \%$ could have been considered sufficient for performing a major liver resection in some cases, in this specific case, an upfront right hepatectomy was considered at high risk for PHLF, due to liver function impairment indicated by a persistently elevated R15 value in the ICG test.

A rescue ALPPS was ultimately considered as a final salvage attempt in this case. The most recognized advantages of ALPPS are the significant increase in FLR volume and the short time interval necessary to achieve it ${ }^{[26]}$. It is an excellent salvage procedure but can also be associated with significant postoperative morbidities and mortality rates ${ }^{[27]}$, probably due to the fact that the fast liver hypertrophy following ALPPS does not necessarily correspond to a rapid liver function improvement. In the current case, however, the further increase in FLR volume was satisfactory.

To reduce the risk of infectious complication following the first step of ALPPS in a right liver already deprived of its portal inflow and venous outflow, we opted for a mini-ALPPS, consisting in a less aggressive 

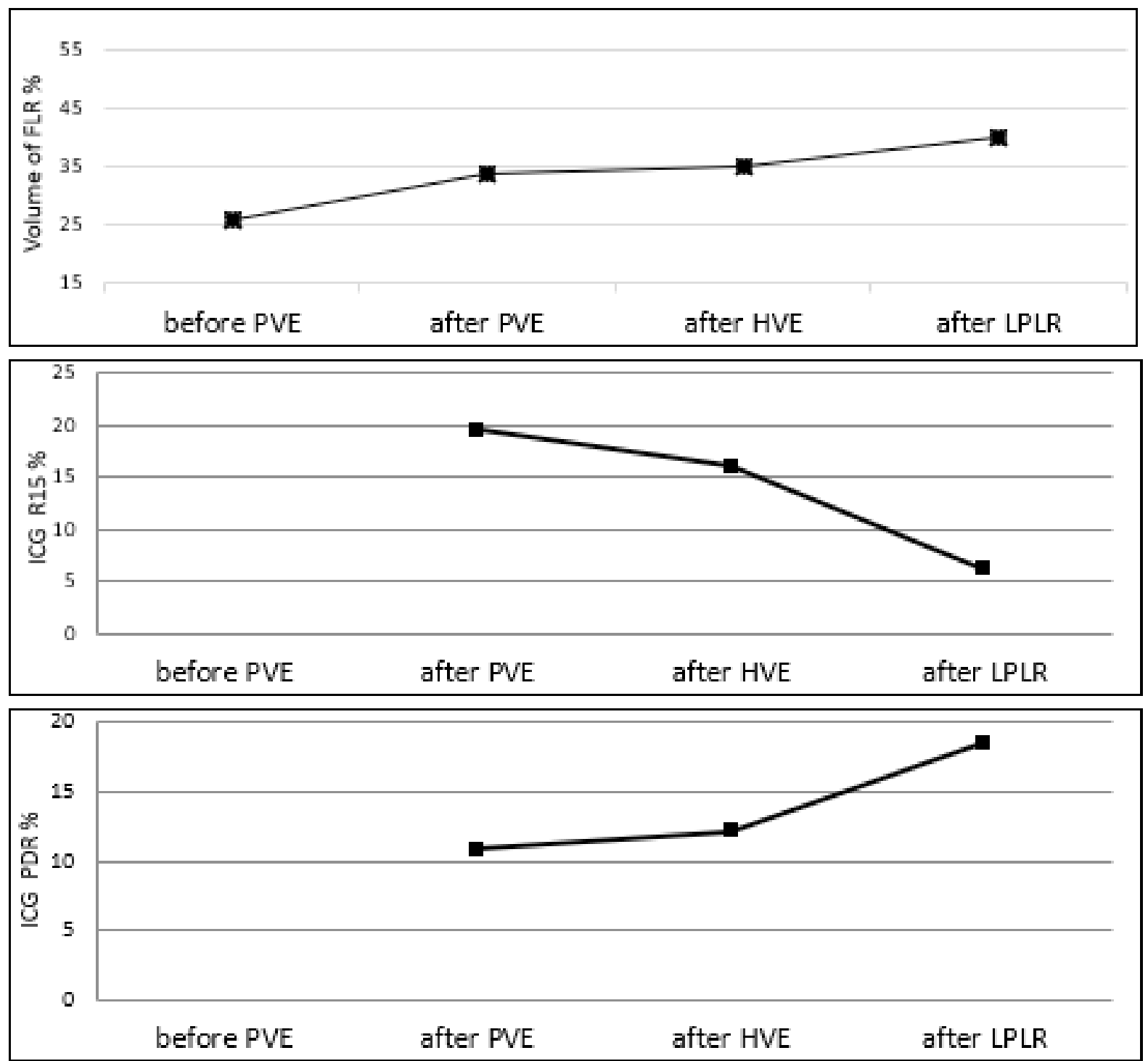

Figure 3. Trend of FLR volume \%, ICG R15\% and ICG PDR \% at diagnosis and after the following steps: right PVE, right HVE and LPLR (first-stage mini-ALPPS) in our clinical case. FLR: future liver remnant; PVE: portal vein embolization; HVE: hepatic vein embolization; LPLR: laparoscopic partial liver resection.

first stage operation during which only a partial hepatic parenchymal transection is performed ${ }^{[28,29]}$. We believe that such approach, reducing the surgical stress related to the first stage operation, may enhance postoperative recovery and may increase the patient's chance of reaching the second stage with a good performance status.

In this case, our approach was successful not only in increasing the FLR [Figure 2] but also in improving the patient's liver function, with a decrease in R15 of $61 \%$ (from 16 to $6.2 \%$ ) and an increase in PDR of $52 \%$ (from $12.2 \%$ to $18.5 \%$ ) [Figure 3]. Following ALPPS first stage, an increase in liver function (measured by ICG test), despite inferior to the increase in FLR volume, has been previously reported in a series of nine patients undergoing ALPPS for colorectal liver metastases ${ }^{[30]}$. In the case presented here, the remarkable decrease in R15\% following laparoscopic partial parenchymal transection probably reflects a boost in the FLR function induced by the further devascularization of the right liver previously deprived of the portal inflow and of the hepatic venous outflow. 
An important aspect of our case is the total laparoscopic approach used in both operations. Laparoscopic liver surgery has been gaining popularity, thanks to many studies confirming its safety and oncological efficacy $^{[31-34]}$, and this has also been proven even when 2-stage hepatectomies are performed ${ }^{[22]}$. In fact, laparoscopic two-stage hepatectomy was considered safe and feasible and without detrimental effects on long-term outcomes in the Southampton guidelines ${ }^{[35]}$.

A recent systematic review comparing available literature on minimally invasive and open ALPPS suggests better short-term outcomes for the minimally invasive approach ${ }^{[33]}$, highlighting, however, that minimally invasive ALPPS requires advanced surgical skills, and as such, it is difficult to reproduce.

In conclusion, this report highlights the importance of the MDT in guiding decision-making and in opening the possibility of integrating different strategies. In addition, this case is a clear call for attempting every possible strategy to enable a radical treatment even in extreme cases. In fact, the multidisciplinary approach for cancer management has been one of the greatest developments in patient's care in the last 2 decades.

Fully laparoscopic ALPPS is a technically challenging procedure; however, it is feasible and safe when performed in centers with advanced experience in laparoscopic liver surgery and may help in reducing the surgical stress both after the first stage ALPPS operation, thus allowing patients to approach the second procedure in a better shape, and after the second stage of ALPPS, enhancing functional postoperative recovery.

\section{DECLARATIONS}

\section{Authors' contributions}

Conceived designed the study: Abu Hilal M

Wrote the manuscript: Malerba V, Zimmitti G

Analyzed the data: Malerba V, Benedetti Cacciaguerra A, Zimmitti G

Edited the video: Benedetti Cacciaguerra A

Mprovided data and critically revised the manuscript: Manzoni A, Sallemi C, Monfardini L, Graffeo M, Bnà C, Natalini G, Zaniboni A, Montresor MC, Codignola C, Garatti M, Abu Hilal M

\section{Availability of data and materials}

Not Applicable.

\section{Financial support and sponsorship}

None.

\section{Conflicts of interest}

All authors declared that there are no conflicts of interest.

\section{Ethical approval and consent to participate}

Not Applicable.

\section{Consent for publication}

Consent form of video and images were taken after patients consent for publication.

\section{Copyright}

(c) The Author(s) 2021. 


\section{REFERENCES}

1. Kishi Y, Abdalla EK, Chun YS, et al. Three hundred and one consecutive extended right hepatectomies: evaluation of outcome based on systematic liver volumetry. Ann Surg 2009;250:540-8.

2. Lang H, Sotiropoulos GC, Brokalaki EI, et al. Left hepatic trisectionectomy for hepatobiliary malignancies. $J$ Am Coll Surg 2006;203:311-21.

3. Adam R, Avisar E, Ariche A, et al. Five-year survival following hepatic resection after neoadjuvant therapy for nonresectable colorectal. Ann Surg Oncol 2001;8:347-53.

4. Ge PL, Du SD, Mao YL. Advances in preoperative assessment of liver function. Hepatobiliary Pancreat Dis Int 2014;13:361-70.

5. Ren Z, Xu Y, Zhu S. Indocyanine green retention test avoiding liver failure after hepatectomy for hepatolithiasis. Hepatogastroenterology 2012;59:782-4.

6. Wakabayashi H, Yachida S, Maeba T, Maeta H. Evaluation of liver function for the application of preoperative portal vein embolization on major hepatic resection. Hepatogastroenterology 2002;49:1048-52.

7. Vauthey JN, Dixon E, Abdalla EK, et al; American Hepato-Pancreato-Biliary Association., Society of Surgical Oncology., Society for Surgery of the Alimentary Tract. Pretreatment assessment of hepatocellular carcinoma: expert consensus statement. HPB (Oxford) 2010;12:289-99.

8. May BJ, Madoff DC. Portal vein embolization: rationale, technique, and current application. Semin Intervent Radiol 2012;29:81-9.

9. van Lienden KP, van den Esschert JW, de Graaf W, et al. Portal vein embolization before liver resection: a systematic review. Cardiovasc Intervent Radiol 2013;36:25-34.

10. Azoulay D, Castaing D, Krissat J, et al. Percutaneous portal vein embolization increases the feasibility and safety of major liver resection for hepatocellular carcinoma in injured liver. Ann Surg 2000;232:665-72.

11. Okabe H, Beppu T, Ishiko T, et al. Preoperative portal vein embolization (PVE) for patients with hepatocellular carcinoma can improve resectability and may improve disease-free survival. J Surg Oncol 2011;104:641-6.

12. Schnitzbauer AA, Lang SA, Goessmann H, et al. Right portal vein ligation combined with in situ splitting induces rapid left lateral liver lobe hypertrophy enabling 2-staged extended right hepatic resection in small-for-size settings. Ann Surg 2012;255:405-14.

13. Lang H, de Santibañes E, Schlitt HJ, et al. 10th Anniversary of ALPPS-Lessons Learned and quo Vadis. Ann Surg 2019;269:114-9.

14. Niekamp AS, Huang SY, Mahvash A, et al. Hepatic vein embolization after portal vein embolization to induce additional liver hypertrophy in patients with metastatic colorectal carcinoma. Eur Radiol 2020;30:3862-8.

15. Wong MC, Jiang JY, Goggins WB, et al. International incidence and mortality trends of liver cancer: a global profile. Sci Rep 2017;7:45846.

16. Poon RT. Optimal initial treatment for early hepatocellular carcinoma in patients with preserved liver function: transplantation or resection? Ann Surg Oncol 2007;14:541-7.

17. Pulitano C, Crawford M, Joseph D, Aldrighetti L, Sandroussi C. Preoperative assessment of postoperative liver function: the importance of residual liver volume. J Surg Oncol 2014;110:445-50.

18. Tustumi F, Ernani L, Coelho FF, et al. Preoperative strategies to improve resectability for hepatocellular carcinoma: a systematic review and meta-analysis. $H P B$ (Oxford) 2018;20:1109-18.

19. Hwang S, Lee SG, Ko GY, et al. Sequential preoperative ipsilateral hepatic vein embolization after portal vein embolization to induce further liver regeneration in patients with hepatobiliary malignancy. Ann Surg 2009;249:608-16.

20. Abdalla EK, Hicks ME, Vauthey JN. Portal vein embolization: rationale, technique and future prospects. Br J Surg 2001;88:165-75.

21. Görgec B, Suhool A, Al-Jarrah R, et al. Surgical technique and clinical results of one- or two-stage laparoscopic right hemihepatectomy after portal vein embolization in patients with initially unresectable colorectal liver metastases: A case series. Int J Surg 2020;77:69-75.

22. Jain G, Parmar J, Mohammed MM, et al. "Stretching the limits of laparoscopic surgery": two-stage laparoscopic liver resection. $J$ Laparoendosc Adv Surg Tech A 2010;20:51-4.

23. Widmann WD, Hales MR, Greenspan RH. The effects of hepatic vein occlusions. Am J Pathol 1962;41:439-54.

24. Hwang S, Ha TY, Ko GY, et al. Preoperative Sequential Portal and Hepatic Vein Embolization in Patients with Hepatobiliary Malignancy. World J Surg 2015;39:2990-8.

25. van Lienden KP, van den Esschert JW, Rietkerk M, et al. Short-term effects of combined hepatic vein embolization and portal vein embolization for the induction of liver regeneration in a rabbit model. J Vasc Interv Radiol 2012;23:962-7.

26. Schadde E, Ardiles V, Robles-Campos R, et al; ALPPS Registry Group. Early survival and safety of ALPPS: first report of the International ALPPS Registry. Ann Surg 2014;260:829-36; discussion 836-8.

27. Dokmak S, Belghiti J. Which limits to the "ALPPS” approach? Ann Surg 2012;256:e6.

28. de Santibañes E, Clavien PA. Playing Play-Doh to prevent postoperative liver failure: the "ALPPS" approach. Ann Surg 2012;255:415-7.

29. Chan KS, Low JK, Shelat VG. Associated liver partition and portal vein ligation for staged hepatectomy: a review. Transl Gastroenterol Hepatol 2020;5:37.

30. Sparrelid E, Jonas E, Tzortzakakis A, et al. Dynamic Evaluation of Liver Volume and Function in Associating Liver Partition and Portal Vein Ligation for Staged Hepatectomy. J Gastrointest Surg 2017;21:967-74.

31. Abu Hilal M, Di Fabio F, Abu Salameh M, Pearce NW. Oncological efficiency analysis of laparoscopic liver resection for primary and metastatic cancer: a single-center UK experience. Arch Surg 2012;147:42-8.

32. Wakabayashi G, Cherqui D, Geller DA, et al. Recommendations for laparoscopic liver resection: a report from the second international consensus conference held in Morioka. Ann Surg 2015;261:619-29.

33. Cipriani F, Shelat VG, Rawashdeh M, et al. Laparoscopic Parenchymal-Sparing Resections for Nonperipheral Liver Lesions, the 
Diamond Technique: Technical Aspects, Clinical Outcomes, and Oncologic Efficiency. J Am Coll Surg 2015;221:265-72.

34. Ciria R, Cherqui D, Geller DA, Briceno J, Wakabayashi G. Comparative Short-term Benefits of Laparoscopic Liver Resection: 9000 Cases and Climbing. Ann Surg 2016;263:761-77.

35. Abu Hilal M, Aldrighetti L, Dagher I, et al. The Southampton Consensus Guidelines for Laparoscopic Liver Surgery: From Indication to Implementation. Ann Surg 2018;268:11-8. 